\title{
Dimensão Psicológica da Qualidade de Vida de Estudantes de Medicina
}

\author{
Psychological Dimension of Quality of Life \\ Among Medical Students
}

Sofia Senna Gonçalves ${ }^{I}$ Annibal Muniz Silvany Neto ${ }^{I}$

\section{PALAVRAS-CHAVE: \\ - Qualidade de Vida; \\ - Estudantes de Medicina; \\ - Educação Médica.}

\section{KEYWORDS:}

- Quality of Life;

- Students, Medical;

- Education, Medical.

\section{Recebido em: 18/03/2012}

Reencaminhado em: 24/06/2013

Aprovado em: 31/07/2013

REVISTA BRASILEIRA DE EDUCAÇÃO MÉDICA

\section{RESUMO}

Objetivos: Avaliar a Dimensão Psicológica da Qualidade de Vida (DPQV) dos estudantes de Medicina da Universidade Federal da Bahia, caracterizar a população segundo variáveis sociodemográficas e epidemiológicas, e identificar possíveis fatores associados. Método: Estudo transversal baseado em questionário autoaplicável com questões da DPQV propostas pela OMS, aplicado aos estudantes do primeiro ao oitavo semestre. Resultados: Os fatores relevantes de aumento da prevalência de baixa DPQV foram: estar cursando o segundo ano; ser do sexo feminino; ser casado(a); morar com os pais versus morar sozinho ou com outras pessoas; renda familiar menor que 15 salários mínimos ou não saber a renda familiar versus renda de mais de 15 salários mínimos; ter escolhido cursar Medicina por motivos não relacionados à aptidão; não dispor de dinheiro suficiente para as necessidades cotidianas; não ter atividades extra-acadêmicas prazerosas; insatisfação com o custo das refeições, a qualidade dos livros na biblioteca e a segurança no ambiente da faculdade; alimentação não saudável; ingerir álcool versus nenhuma ingestão; e fazer ou ter feito uso de drogas ilícitas. Conclusão: Os resultados sugerem influência do curso de Medicina na DPQV.

\begin{abstract}
Objective: To evaluate the Psychological Dimension of Quality of Life (PDQOL) of medical students at the Federal University of Bahia, examining the sample population in terms of sociodemographic and epidemiological variables and identifying associated factors. Method: A cross-sectional study based on a self-administered questionnaire incorporating questions of PDQOL proposed by the WHO, applied to 1st to 8th semester students. Results: The relevant factors identified for the increased prevalence of low PDQOL were: being a second year medical student, female, married, living with parents versus living alone or with others; having a family income of less than 15 minimum wages or not knowing the family income versus an income of more than 15 minimum wages; having chosen medicine for reasons not related to ability; not having enough money for everyday needs; not practicing any pleasurable extracurricular activities; being dissatisfied with the cost of meals, the quality of the books in the library and safety on campus; unhealthy diet; drinking alcohol versus being teetotal; and using or having used illicit drugs. Conclusion: The results suggest that medical school does have an influence on PDQOL.
\end{abstract}




\section{INTRODUÇÃO}

A importância de avaliar a qualidade de vida (QV) dos indivíduos foi reconhecida mais amplamente nos anos 1990, quando a Organização Mundial de Saúde (OMS) propôs uma definição e uma forma de avaliação deste conceito. Para a OMS, qualidade de vida é definida como "a percepção do indivíduo de sua posição na vida no contexto da cultura e sistema de valores nos quais ele vive e em relação aos seus objetivos, expectativas, padrões e preocupações" ${ }^{\prime 1}$.

A qualidade de vida é um elemento-chave no processo saúde-doença de uma população. Seu conceito traz a natureza biopsicossocial do indivíduo e considera aspectos físicos, psicológicos, sociais, ambientais e a subjetividade da percepção do indivíduo sobre a própria vida. A medida de QV é um fato irreversível, que, provavelmente, vai pertencer ao nosso universo, da mesma forma que a ecografia ${ }^{2}$. O questionário de avaliação da dimensão psicológica da qualidade de vida da OMS (Whoqol-Bref) avalia os seguintes aspectos: sentimentos positivos, pensar e aprender, autoestima, imagem corporal, sentimentos negativos e espiritualidade ${ }^{1}$. O número de estudos que utilizaram este questionário vem crescendo ao longo dos anos, sendo o Brasil um dos destaques em quantidade de publicações ${ }^{3}$.

Várias características do curso de Medicina o tornam potencialmente atuante na QV dos estudantes, como alta carga horária, densidade de informações e cobrança dentro e fora do ambiente acadêmico. Já foram identificados fatores estressantes na educação médica que levam a consequências na saúde dos estudantes ${ }^{4}$.

Pesquisas em populações de estudantes de Medicina têm mostrado diversas alterações em sua QV em proporções maiores do que as observadas na população geral, destacando-se maior prevalência de transtornos mentais comuns ${ }^{5,6}$, menor QV em relação a estudantes de Direito ${ }^{7}$, alta prevalência de episódios depressivos maiores ${ }^{8}$, de transtornos mentais comuns $^{9}$ e de distúrbios do sono ${ }^{10}$.

Considerando-se os sintomas depressivos em geral, independentemente de seu grau, verificou-se uma frequência de $79 \%$ entre estudantes de Medicina ${ }^{11}$. Estes ainda apresentam altos escores de traço e estado de ansiedade ${ }^{12}$, além de estudos apontarem aumento do uso de tranquilizantes após o início do curso médico ${ }^{13}$ e prevalência de sintomas de estresse nos estudantes de Medicina de 49,7\%, sendo que 73,5\% deles consideraram suas atividades na faculdade como fonte estressora ${ }^{14}$, representando uma relação causal apontada pelos próprios alunos.

Alterações no domínio psicológico de um indivíduo são, ainda, gênese em potencial de desenvolvimento de transtornos psiquiátricos e de ideação suicidas. Em revisão da litera- tura, verificou-se que em toda parte do mundo a proporção de suicídio na população médica era superior à da população geral $^{15}$ e que, após os acidentes, o suicídio era a causa mais comum de morte entre os estudantes de Medicina ${ }^{16}$.

Não se encontra na literatura um amplo resultado de avaliações da QV em estudantes de Medicina por meio do questionário da OMS. Entretanto, há uma preocupação em avaliar o estado psíquico desses estudantes e sua relação com o início e a progressão do curso, havendo diversos estudos de avaliação de estresse, distúrbios do sono e depressão.

A avaliação da QV dos estudantes de Medicina ganha relevância não somente por seu impacto durante o curso, mas também em uma perspectiva de conhecimento, discussão e saneamento de alguns fatores predisponentes de baixa qualidade de vida dos próprios médicos, os quais poderão interferir na sua atuação profissional futura e, consequentemente, na funcionalidade da saúde no País. A avaliação do modo como o curso de Medicina afeta a dimensão psicológica da QV dos estudantes pode servir para aprimorar os pontos mais críticos na formação dos médicos a fim de melhorar a saúde mental e a formação desses profissionais.

Com base na hipótese de que diversas características do curso de Medicina favorecem a diminuição da qualidade de vida de seus graduandos, o presente estudo objetiva avaliar a dimensão psicológica da qualidade de vida (DPQV) dos estudantes de Medicina da Universidade Federal da Bahia (UFBA) e identificar e avaliar possíveis fatores associados, além de caracterizar a população estudada segundo variáveis sociodemográficas e epidemiológicas.

\section{MÉTODOS}

Foi realizado um estudo transversal exploratório, no qual se incluíram todos os estudantes que cursavam o final do primeiro ao oitavo semestre, além dos recém-ingressos na faculdade no semestre seguinte ao início da coleta de dados. Estes últimos foram incluídos no estudo para servir como população de referência sem influência de fatores relacionados à faculdade. Os estudantes do internato não foram incluídos por dificuldades operacionais e por estarem em momento distinto de formação acadêmica, com particularidades que poderiam impedir comparações adequadas.

A primeira parte do questionário foi elaborada pelos autores com questões de caráter sociodemográfico, abordando alguns dos principais aspectos potencialmente envolvidos na DPQV do estudante. A segunda parte corresponde às perguntas relativas à DPQV contidas no questionário World Health Organization Quality of Life - Bref (Whoqol-Bref). O projeto de pesquisa foi aprovado pelo Comitê de Éti- 
ca Médica da Maternidade Climério de Oliveira, por meio do Parecer/Resolução $n^{\circ}$ 046/2010 e registro $n^{\circ}$ 036/10. Realizou-se um pré-teste, no qual um estudante de cada semestre pesquisado respondeu ao questionário, sugerindo modificações, que foram levadas em conta para seu aperfeiçoamento final.

A coleta de dados ocorreu em dois momentos. Primeiramente, durante os últimos 15 dias do período letivo do semestre 2010.2, correspondentes ao final de novembro e início de dezembro, foram aplicados os questionários em todos os estudantes que cursavam do primeiro ao oitavo semestre naquele momento. Os questionários e os termos de consentimento foram entregues aos alunos em sala com a devida permissão dos professores, preferencialmente em horários de provas, para diminuir a perda por faltas, e devolvidos no mesmo dia. O segundo momento de coleta consistiu na aplicação do mesmo questionário durante o semestre de 2011.1 apenas aos estudantes recém-ingressos na faculdade, durante a primeira quinzena de aulas do curso. A aplicação precoce dos questionários nesse momento foi proposital, a fim de abordar os recém-ingressos antes que sofressem importante influência da faculdade e seu meio, contrapondo-se à abordagem anterior, feita em todos os estudantes no final de seus respectivos semestres e que visava obter informações sobre as vivências referentes a cada um dos diferentes semestres, para fins comparativos. Dois alunos de graduação, incluindo a autora, participaram da coleta.

Os dados obtidos por meio dos questionários foram digitados e processados com uso do programa Statistical Package for the Social Sciences (SPSS), versão 17. As questões correspondentes à DPQV, retiradas do questionário Whoqol-Bref, foram processadas de acordo com o escore sugerido pela OMS. A OMS não sugere um ponto de corte para este escore. Dessa forma, a mediana dos escores obtidos $(66,67)$ foi usada como ponto de corte entre baixa e alta DPQV, tendo sido considerados com alta DPQV aqueles com escore igual ou superior a 66,7, e com baixa DPQV aqueles com escore abaixo deste valor. A variável DPQV foi então considerada a variável dependente do estudo, e as demais variáveis envolvidas foram consideradas independentes.

A abordagem estatística dos dados compreendeu três etapas: descrição da população estudada, usando-se procedimentos usuais de estatística descritiva, tais como: cálculo de frequências, de medidas de tendência central e de dispersão, e elaboração de diagramas; análise bivariável para identificar possíveis associações estatísticas entre as variáveis independentes e a variável dependente, utilizando-se o cálculo de razões de médias e razões de prevalência; análise multivariável por meio da regressão logística múltipla, seguindo-se alguns procedimentos recomendados por Hosmer e Lemeshow ${ }^{17}$. A avaliação da escala mais adequada para expressão de variáveis contínuas na modelagem foi feita por meio de análise gráfica e do método dos polinômios fracionários, conforme sugestão desses mesmos autores ${ }^{17}$.

Como não houve amostragem, não foram utilizados os procedimentos de inferência estatística em nenhuma dessas etapas de abordagem estatística. Assim, na análise bivariável, a importância estatística de cada variável foi avaliada usando-se como critério um excesso de prevalência maior do que $30 \%$. Este foi o mesmo critério utilizado para manutenção de variáveis no modelo final da análise de regressão logística múltipla. Contudo, na pré-seleção de variáveis para essa modelagem, o critério empregado foi um excesso de prevalência de $20 \%$, para evitar a remoção precoce de variáveis possivelmente importantes. A qualidade do ajuste do modelo foi feita por meio de análise de resíduos e de influência ${ }^{17}$.

\section{RESULTADOS}

Dos 637 estudantes matriculados do primeiro ao oitavo semestre, 564 responderam ao questionário, correspondendo a uma perda de $11,5 \%$, e, entre os 80 recém-ingressos de 2011.1, 63 responderam ao questionário. A maioria dos estudantes (58,9\%) e dos recém-ingressos (50,8\%) era do sexo masculino. As idades dos estudantes variaram de 17 a 40 anos, quase $90 \%$ tinham entre 17 e 25 anos, e a média foi de 22 anos com desvio padrão de 3,62. As idades dos recém-ingressos variaram de 17 a 36 anos, e a média foi de 20,22 anos, com desvio padrão de 3,37 anos. Quase metade (48\%) dos estudantes e a maioria dos recém-ingressos (52,4\%) eram naturais de Salvador. A religião mais prevalente nos dois grupos foi católica romana. Cerca de $15 \%$ dos estudantes e $17 \%$ dos recém-ingressos eram ateus ou agnósticos.

A maior parte dos alunos (53\%) e dos recém-ingressos $(49,2 \%)$ morava com os pais. Quase metade dos estudantes $(49 \%)$ referiu renda familiar mensal de até dez salários mínimos, e a maior parte $(30,9 \%)$ dos recém-ingressos informou renda entre 11 e 15 salários mínimos. Apenas $11,4 \%$ dos estudantes tinham outra ocupação. Destes, $61,4 \%$ tinham trabalho fixo e $17,5 \%$ eram professores. Menos de $5 \%$ dos recém-ingressos tinham outra ocupação. Cerca de $23 \%$ dos estudantes e $16 \%$ dos recém-ingressos julgaram não ter dinheiro suficiente para suas necessidades cotidianas. Mais de $40 \%$ dos estudantes e cerca de $27 \%$ dos recém-ingressos não desempenhavam regularmente atividades extra-acadêmicas que lhes dessem prazer. 
A maioria dos estudantes (59,3\%) não considerou sua alimentação como saudável, devido, sobretudo, à falta de tempo $(73,2 \%)$. A maioria dos recém-ingressos considerou sua alimentação como saudável (60,3\%). A maior parte dos estudantes $(59,1 \%)$ e dos recém-ingressos $(61,3 \%)$ não praticava atividade física. $\mathrm{O}$ tempo semanal entre os estudantes que praticavam variou de 30 minutos a 14 horas, com média de 4 horas e 22 minutos e desvio padrão de 2 horas e 26 minutos. Entre os recém-ingressos, o tempo variou de 20 minutos a 10 horas/semana, com média de 4 horas e 40 minutos e desvio padrão de cerca de 2 horas e 32 minutos.

A maioria dos alunos $(70,7 \%)$ e dos recém-ingressos $(75,8 \%)$ nunca havia fumado ou tinha parado de fumar havia mais de dez anos. A duração do tabagismo variou de três meses a dez anos, com média de dois anos e dez meses, com desvio padrão de dois anos e quatro meses, e mediana de dois anos. Aproximadamente um terço dos estudantes e dos recém-ingressos tomava bebida alcoólica de uma a três vezes por mês. Cerca de $16 \%$ dos estudantes e 14,5\% dos recém-ingressos haviam feito uso passado de drogas ilícitas. Mais de $5 \%$ dos estudantes faziam uso atual. Dos que já haviam usado, quase $37 \%$ usaram a primeira vez após o início da faculdade. Nos últimos seis meses, a droga ilícita mais usada foi a maconha, em ambos os grupos.

Os mais frequentes motivos de escolha do curso de Medicina foram "aptidão" e "desejo de ajudar os outros". Quase $40 \%$ pensavam ou haviam pensado em abandonar o curso, principalmente devido ao cansaço e à sobrecarga (49,5\%). A maioria julgou seu desempenho acadêmico como bom $(52,4 \%)$ e $6,4 \%$ o julgaram como ruim ou péssimo. Em relação ao ambiente da faculdade, a maioria dos estudantes não estava satisfeita com a qualidade $(94,4 \%)$ e o custo $(91 \%)$ das refeições disponíveis, assim como com a quantidade $(93,2 \%)$ e a qualidade $(86,9 \%)$ dos livros na biblioteca, e a segurança $(80,8 \%)$.

Quase 30\% dos estudantes e apenas 6,5\% dos recém-ingressos aproveitavam a vida "nada ou muito pouco". Para a pergunta "em que medida sua vida tem sentido?", a maioria dos estudantes $(77,5 \%)$ e dos recém-ingressos $(81,7 \%)$ respondeu "bastante" ou "extremamente". Quase 5\% dos estudantes responderam "nada" ou "muito pouco". A maioria (51,7\%) dos estudantes conseguia se concentrar "mais ou menos" e a maioria dos recém-ingressos conseguia se concentrar "bastante" ou "extremamente" (65\%). A maioria dos alunos (62,1\%) e dos recém-ingressos $(59,7 \%)$ era muito ou completamente capaz de aceitar sua aparência física. Cerca de 15\% dos estudantes e apenas $4,8 \%$ dos recém-ingressos estavam insatisfeitos ou muito insatisfeitos consigo mesmos. A maioria dos estudantes
$(56,4 \%)$ tinha sentimentos negativos algumas vezes e cerca de $37 \%$ deles os tinham frequentemente ou muito frequentemente, contra 16\% dos recém-ingressos (Tabela 1).

TABELA 1

Resultados da aplicação do questionário de Dimensão Psicológica da Qualidade de Vida (OMS) nos estudantes do primeiro ao oitavo semestre e recém-ingressos da FMB-UFBA, Salvador, 2010/2011

\begin{tabular}{|c|c|c|c|c|}
\hline \multirow[t]{2}{*}{ Variável } & \multicolumn{2}{|c|}{$\begin{array}{l}\text { Estudantes } \\
\text { (1a ao } 8^{\circ} \\
\text { semestre) }\end{array}$} & \multicolumn{2}{|c|}{$\begin{array}{l}\text { Recém- } \\
\text { ingressos }\end{array}$} \\
\hline & $\mathbf{N}$ & $\%$ & $\mathbf{N}$ & $\%$ \\
\hline \multicolumn{5}{|l|}{ O quanto aproveita a vida } \\
\hline Nada & 23 & 4,1 & 0 & 0,0 \\
\hline Muito pouco & 144 & 25,8 & 4 & 6,5 \\
\hline Mais ou menos & 234 & 41,9 & 24 & 38,7 \\
\hline Bastante & 145 & 25,9 & 31 & 50,0 \\
\hline Extremamente & 13 & 2,3 & 3 & 4,8 \\
\hline \multicolumn{5}{|l|}{ Em que medida a vida tem sentido } \\
\hline Muito pouco & 5 & 0,9 & 0 & 0,0 \\
\hline Mais ou menos & 22 & 4,0 & 1 & 1,7 \\
\hline Bastante & 98 & 17,6 & 10 & 16,7 \\
\hline Extremamente & 226 & 40,6 & 21 & 35,0 \\
\hline 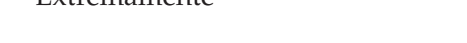 & 205 & 36,9 & 28 & 46,7 \\
\hline \multicolumn{5}{|l|}{ Quanto consegue se concentrar } \\
\hline Nada & 13 & 2,3 & 0 & 0,0 \\
\hline Muito pouco & 109 & 19,6 & 4 & 6,7 \\
\hline Mais ou menos & 288 & 51,7 & 17 & 28,3 \\
\hline Bastante & 134 & 24,1 & 35 & 58,3 \\
\hline Extremamente & 13 & 2,3 & 4 & 6,7 \\
\hline \multicolumn{5}{|l|}{ Capaz de aceitar a aparência física } \\
\hline Nada & 32 & 5,7 & 4 & 6,5 \\
\hline Muito pouco & 179 & 32,0 & 20 & 32,3 \\
\hline Médio & 220 & 39,4 & 16 & 25,8 \\
\hline $\begin{array}{l}\text { Muito } \\
\text { Completamente }\end{array}$ & 127 & 22,7 & 21 & 33,9 \\
\hline Satisfação consigo mesmo & 13 & 2,3 & 0 & 0,0 \\
\hline Muito insatisfeito & 72 & 12,9 & 3 & 4,8 \\
\hline Insatisfeito & 119 & 21,3 & 10 & 16,1 \\
\hline Nem satisfeito nem insatisfeito & 277 & 49,6 & 33 & 53,2 \\
\hline Satisfeito & 78 & 14,0 & 16 & 25,8 \\
\hline Muito satisfeito & & & & \\
\hline \multicolumn{5}{|l|}{ Frequência de sentimentos negativos } \\
\hline Sempre & 11 & 2,0 & 0 & 0,0 \\
\hline Muito frequentemente & 59 & 10,6 & 1 & 1,6 \\
\hline Frequentemente & 149 & 26,8 & 9 & 14,8 \\
\hline Algumas vezes & 314 & 56,4 & 49 & 80,3 \\
\hline Nunca & 24 & 4,3 & 2 & 3,3 \\
\hline
\end{tabular}


A Tabela 2 contém as variáveis independentes dicotômicas estudadas que apresentaram excesso de prevalência de baixa DPQV igual ou superior a 30\%. Foram elas: falta de apoio da família; relação ruim com os pais, com os irmãos, com os amigos ou com o(a) parceiro(a); dinheiro insuficiente para as atividades cotidianas; não desempenhar atividades prazerosas extra-acadêmicas; insatisfação com o custo das refeições, com a qualidade e quantidade de livros na biblioteca ou com a segurança na faculdade; alimentação não saudável; não praticar atividade física; ter iniciado uso de drogas ilícitas após ingressar na faculdade e ter usado anfetamina ou tranquilizante nos últimos seis meses. As demais variáveis independentes dicotômicas estudadas, cujos excessos de prevalência não chegaram a 30\%, foram: sexo, ter ou não outra ocupação além de estudante, ter ou não dívidas que estejam preocupando, uso ou não de maconha nos últimos seis meses, uso ou não de outra droga nos últimos seis meses e grau de satisfação com a qualidade das refeições disponíveis no ambiente da faculdade, tendo esta última atingido um excesso de prevalência limítrofe de $29 \%$.

A média de idade dos estudantes com baixa DPQV foi de 22,10 anos ( $\mathrm{DP}=3,07$ ), pouco maior do que a média entre os estudantes com alta DPQV, que foi de 21,91 (DP = 2,98). A média de tempo de atividade física entre aqueles com baixa DPQV foi de 249,38 minutos (DP $=142,65$ ), sendo $8,7 \%$ menor do que a média entre os estudantes com alta DPQV, que foi de 273,09 minutos ( $\mathrm{DP}=149,21)$. Os recém-ingressos não foram incluídos na análise bivariável, pois, considerando que ainda não haviam iniciado o curso de Medicina, não haviam sofrido influência de muitas das variáveis estudadas.

Os resultados da análise multivariável estão apresentados na Tabela 3. Encontraram-se como prováveis fatores de aumento da prevalência de baixa DPQV: o segundo ano do curso de Medicina, sexo feminino, ser casado(a), morar com os pais versus morar sozinho ou com outros (não parentes ou amigos), renda familiar menor que 15 salários mínimos ou não saber a renda familiar versus renda de mais de 15 salários mínimos, ter escolhido cursar Medicina pelo mercado de trabalho ou por outros motivos não relacionados à aptidão, não dispor de dinheiro suficiente para as necessidades cotidianas, não ter atividades extra-acadêmicas que deem prazer, estar insatisfeito com o custo das refeições disponíveis no ambiente da faculdade, estar insatisfeito com a qualidade de livros disponíveis na biblioteca da faculdade, estar insatisfeito com a segurança no ambiente da faculdade, não ter uma alimentação saudável, não ingerir álcool versus ingerir com frequência de pelo menos uma vez por mês, e uso atual ou passado de droga ilícita.
TABELA 2

Análise bivariável entre algumas variáveis independentes discretas estudadas e a dimensão psicológica da qualidade de vida, em estudantes do primeiro ao oitavo semestre da FMB-UFBA, Salvador, 2010

\begin{tabular}{|c|c|c|c|c|c|}
\hline \multirow{3}{*}{ Variável } & \multicolumn{4}{|c|}{$\begin{array}{l}\text { Dimensão Psicológica da } \\
\text { Qualidade de Vida }\end{array}$} & \multirow{3}{*}{ RP } \\
\hline & \multicolumn{2}{|c|}{ Baixa } & \multicolumn{2}{|c|}{ Alta } & \\
\hline & $\mathbf{N}$ & $\%$ & $\mathbf{N}$ & $\%$ & \\
\hline \multicolumn{6}{|l|}{ Apoio da família } \\
\hline Não & 8 & 80,0 & 2 & 20,0 & \multirow[t]{2}{*}{1,57} \\
\hline Sim & 275 & 50,8 & 266 & 49,2 & \\
\hline \multicolumn{6}{|l|}{ Relação com os pais } \\
\hline Ruim & 14 & 73,7 & 5 & 26,3 & \multirow[t]{2}{*}{1,46} \\
\hline Boa & 265 & 50,5 & 260 & 49,5 & \\
\hline \multicolumn{6}{|c|}{ Relação com os irmãos } \\
\hline Ruim & 19 & 74,4 & 8 & 29,6 & \multirow[t]{2}{*}{1,49} \\
\hline Boa & 242 & 50,0 & 242 & 50,0 & \\
\hline \multicolumn{6}{|c|}{ Relação com os amigos } \\
\hline Ruim & 11 & 100,0 & 0 & 0,0 & \multirow[t]{2}{*}{1,99} \\
\hline Boa & 271 & 50,3 & 268 & 49,7 & \\
\hline \multicolumn{6}{|l|}{$\begin{array}{l}\text { Relação com o(a) } \\
\text { parceiro(a) }\end{array}$} \\
\hline Ruim & 7 & 87,5 & 1 & 12,5 & \multirow[t]{2}{*}{1,99} \\
\hline Boa & 139 & 44,0 & 177 & 56,0 & \\
\hline \multicolumn{6}{|c|}{$\begin{array}{l}\text { O dinheiro é suficiente } \\
\text { para atividades cotidianas? }\end{array}$} \\
\hline Não & 91 & 71,7 & 36 & 28,3 & \multirow{2}{*}{1,58} \\
\hline Sim & 192 & 45,3 & 232 & 54,7 & \\
\hline \multirow{2}{*}{\multicolumn{6}{|c|}{$\begin{array}{l}\text { Desempenha regularmente } \\
\text { atividades prazerosas não } \\
\text { acadêmicas? }\end{array}$}} \\
\hline & & & & & \multirow[t]{3}{*}{1,76} \\
\hline Não & 160 & 68,4 & 74 & 31,6 & \\
\hline Sim & 123 & 38,8 & 194 & 61,2 & \\
\hline \multicolumn{6}{|c|}{$\begin{array}{l}\text { Custo das refeições } \\
\text { disponíveis no ambiente } \\
\text { da faculdade }\end{array}$} \\
\hline Insatisfeito & 261 & 52,5 & 236 & 47,5 & \\
\hline Satisfeito & 18 & 36,0 & 32 & 64,0 & 1,46 \\
\hline \multicolumn{6}{|c|}{$\begin{array}{l}\text { Quantidade de livros } \\
\text { disponíveis na biblioteca }\end{array}$} \\
\hline da faculdade & & & & & 1,53 \\
\hline Insatisfeito & 267 & 52,4 & 243 & 47,6 & \\
\hline Satisfeito & 13 & 34,2 & 25 & 65,8 & \\
\hline \multicolumn{6}{|c|}{$\begin{array}{l}\text { Qualidade dos livros } \\
\text { disponíveis na biblioteca }\end{array}$} \\
\hline da faculdade & & & & & 1,54 \\
\hline Insatisfeito & 254 & 53,4 & 222 & 46,6 & \\
\hline Satisfeito & 25 & 34,7 & 47 & 65,3 & \\
\hline \multicolumn{6}{|c|}{$\begin{array}{l}\text { Segurança no ambiente da } \\
\text { faculdade }\end{array}$} \\
\hline Insatisfeito & 239 & 53,8 & 205 & 46,2 & 1,36 \\
\hline Satisfeito & 42 & 39,6 & 64 & 60,4 & \\
\hline \multicolumn{6}{|l|}{$\begin{array}{l}\text { Tem alimentação } \\
\text { saudável? }\end{array}$} \\
\hline Não & 191 & 58,6 & 135 & 41,4 & 1,48 \\
\hline Sim & 89 & 39,6 & 136 & 60,4 & \\
\hline
\end{tabular}




\begin{tabular}{|c|c|c|c|c|c|}
\hline \multirow{3}{*}{ Variável } & \multicolumn{4}{|c|}{$\begin{array}{l}\text { Dimensão Psicológica da } \\
\text { Qualidade de Vida }\end{array}$} & \multirow{3}{*}{$\mathbf{R P}$} \\
\hline & \multicolumn{2}{|c|}{ Baixa } & \multicolumn{2}{|c|}{ Alta } & \\
\hline & $\mathbf{N}$ & $\%$ & $\mathbf{N}$ & $\%$ & \\
\hline \multicolumn{6}{|c|}{$\begin{array}{l}\text { Pratica alguma atividade } \\
\text { física? }\end{array}$} \\
\hline Não & 197 & 60,2 & 130 & 39,8 & 1,60 \\
\hline Sim & 84 & 37,5 & 140 & 62,5 & \\
\hline \multicolumn{6}{|c|}{$\begin{array}{l}\text { Se usou droga, a primeira } \\
\text { vez foi antes ou depois da } \\
\text { faculdade? }\end{array}$} \\
\hline Depois & 25 & 61,0 & 16 & 39,0 & 1,1, \\
\hline Antes & 27 & 39,7 & 41 & 60,3 & \\
\hline \multicolumn{6}{|c|}{$\begin{array}{l}\text { Nos últimos seis meses } \\
\text { usou anfetamina? }\end{array}$} \\
\hline Sim & 12 & 75,0 & 4 & 25,0 & 1,49 \\
\hline Não & 269 & 50,4 & 265 & 49,6 & \\
\hline \multicolumn{6}{|c|}{$\begin{array}{l}\text { Nos últimos seis meses } \\
\text { usou tranquilizante? }\end{array}$} \\
\hline Sim & 24 & 80,0 & 6 & 20,0 & 1,62 \\
\hline Não & 254 & 49,4 & 260 & 50,6 & \\
\hline
\end{tabular}

$R P=$ Razão de Prevalências

TABELA 3

Resultados do modelo final da análise multivariável de algumas variáveis independentes estudadas em relação à dimensão psicológica da qualidade de vida, em estudantes do primeiro ao oitavo semestre de

Medicina da FMB-UFBA, Salvador, 2010

\begin{tabular}{|c|c|}
\hline Variáveis & RC \\
\hline \multicolumn{2}{|l|}{ Ano de curso } \\
\hline $3^{\circ} \times 1^{\circ}$ & 0,73 \\
\hline $4^{\circ} \times 1^{\circ}$ & 0,69 \\
\hline Sexo & \\
\hline Feminino $\mathrm{x}$ masculino & 1,59 \\
\hline $\begin{array}{l}\text { Estado civil } \\
\quad \text { Solteiro x casado }\end{array}$ & 0,43 \\
\hline $\begin{array}{l}\text { Moradia } \\
\text { Sozinho x com pais } \\
\text { Outros x com pais }\end{array}$ & $\begin{array}{l}0,63 \\
0,56\end{array}$ \\
\hline $\begin{array}{l}\text { Renda familiar (em número de salários mínimos) } \\
\text { Não sabe x mais de } 15 \\
\text { De } 1 \text { a } 5 \text { x mais de } 15 \\
\text { De } 6 \text { a } 10 \text { x mais de } 15 \\
\text { De } 11 \text { a } 15 \text { x mais de } 15\end{array}$ & $\begin{array}{l}2,03 \\
1,62 \\
1,93 \\
1,73\end{array}$ \\
\hline $\begin{array}{l}\text { Principal motivo da escolha por Medicina } \\
\text { Mercado de trabalho x aptidão ou motivos relacionados } \\
\text { Outro ou mistura x aptidão ou motivos relacionados }\end{array}$ & $\begin{array}{l}1,84 \\
1,79\end{array}$ \\
\hline $\begin{array}{l}\text { Dinheiro para necessidades cotidianas } \\
\text { Insuficiente } x \text { suficiente }\end{array}$ & 1,99 \\
\hline $\begin{array}{l}\text { Atividades prazerosas extra-acadêmicas } \\
\text { Não x sim }\end{array}$ & 2,13 \\
\hline
\end{tabular}

\begin{tabular}{lc}
\hline Variáveis & RC \\
\hline $\begin{array}{l}\text { Custo da refeição no ambiente da faculdade } \\
\quad \text { Insatisfeito x satisfeito }\end{array}$ & 1,60 \\
Qualidade de livros na biblioteca & 2,03 \\
$\quad$ Insatisfeito x satisfeito & \\
Segurança na faculdade & 1,34 \\
$\quad$ Insatisfeito x satisfeito & \\
Alimentação saudável & 1,44 \\
$\quad$ Não x sim & \\
Frequência de ingestão de álcool nos últimos 12 meses & 0,64 \\
$\quad$ Pelo menos uma vez por mês x nada & 0,55 \\
$\quad$ Se embriaga ao menos uma vez por mês x nada & \\
Uso de droga ilícita & 1,70 \\
$\quad$ Usou no passado x nunca usou & 2,25 \\
$\quad$ Ainda usa x nunca usou &
\end{tabular}

$R C=$ Razão de Chances

\section{DISCUSSÃO}

A expressão Qualidade de Vida abrange muitos significados, que refletem conhecimentos, experiências e valores de indivíduos e coletividades ${ }^{18}$. Uma alta ou baixa QV sofre influência de incontáveis variáveis, que são diferentes em relação a cada pessoa, tornando difícil a avaliação de todas as possíveis variáveis envolvidas. A população estudada, no entanto, constitui um grupo específico de pessoas que compartilham certas características e estão sob influência de alguns fatores comuns que podem ser avaliados a fim de elucidar o quanto cada um deles contribui para a DPQV deste grupo.

$\mathrm{O}$ ano de curso mostrou ter uma importante influência na QV do grupo estudado, estando de acordo com resultados de estudo prévio ${ }^{19}$. O segundo ano foi aquele que esteve mais relacionado à baixa QV no estudante de Medicina da UFBA. Há muitas características referentes à faculdade e ao curso que podem estar relacionadas a este achado, como carga horária ${ }^{20}$, número de componentes curriculares e aulas ${ }^{21}$ e organização do currículo ${ }^{22,23}$.

Estas características podem variar de forma importante entre as diferentes faculdades. Na Faculdade de Medicina da Bahia, o terceiro semestre contém uma das maiores cargas horárias e é considerado entre os alunos como um dos semestres mais difíceis. A administração do tempo de forma que seja possível conciliar provas e aulas representa uma das principais angústias vividas durante o curso de Medicina ${ }^{24}$. O segundo ano é também um ano de transição, quando os estudantes começam a ter contato com pacientes. Esta fase de transição já foi identificada, em estudos prévios, como de maior risco para instabilidade emocional ${ }^{25,26}$ e para baixa $\mathrm{QV}^{19,21}$. Ao comparar os anos entre si, nota-se uma tendência à redução da DPQV no segundo ano de curso, com melhora progressiva até o quarto 
ano. É possível que, a partir do terceiro ano, o estudante tenda a encontrar mecanismos de adaptação para a realidade do curso, a reformular prioridades e a se adequar às exigências de sua formação acadêmica, o que progride até o quarto ano. Nessa etapa, a vivência do estudante no curso deve ficar mais próxima da vivência real do profissional médico, as matérias são mais práticas e é provável que o estudante consiga se enxergar melhor na profissão. Nesse momento, aqueles que não se identificaram com o curso provavelmente já o abandonaram, e aqueles que estão cursando foram os que encontraram motivação para superar as dificuldades que sentiram e devem estar mais à vontade com sua escolha.

O sexo feminino foi importante fator de aumento de prevalência de baixa DPQV. Estudos anteriores têm mostrado que mulheres são mais susceptíveis ao estresse ${ }^{26,27}$, e esta relação foi encontrada em estudos feitos em outras faculdades de Medicina no Brasil ${ }^{19,21}$ e nos Estados Unidos ${ }^{28}$. Além disso, estudos mostram maior prevalência de sintomas depressivos em estudantes de Medicina do sexo feminino ${ }^{25,29}$.

De acordo com Allardt, ser casado é um fator de estabilidade emocional e social ${ }^{30}$, o que deve se refletir na $\mathrm{QV}$ do indivíduo. Estudo brasileiro feito com estudantes do curso de Educação Física mostrou melhor QV naqueles que eram casa$\operatorname{dos}^{21}$. No presente trabalho, entretanto, os solteiros apresentaram maior DPQV do que os casados. Os estudantes do curso de Medicina se caracterizam por serem jovens e disporem de pouco tempo livre devido à grande carga horária acadêmica, $\mathrm{o}$ que pode explicar em parte a baixa prevalência de indivíduos casados encontrada. Dessa forma, a responsabilidade de manter financeiramente uma família e dispor de tempo para isto pode representar um fator estressor em meio a outras responsabilidades relacionadas à formação acadêmica.

Morar com pessoas que não fossem os pais, outros familiares ou amigos mostrou-se fator de aumento de prevalência de alta DPQV, quando comparado a morar com os pais. É possível que isto seja reflexo do desejo de individualidade e independência próprio do início da vida adulta e não deve representar uma perda importante do apoio familiar e social. Outro estudo feito com estudantes de Medicina, entretanto, não encontrou diferença significativa na QV dos estudantes que residiam com ou sem os familiares ${ }^{19}$.

Os estudantes que tinham renda familiar mensal acima de 15 salários mínimos apresentaram DPQV significativamente maior do que os que tinham renda familiar abaixo disso. A relação com renda familiar não foi encontrada em estudo prévio em outra faculdade de Medicina brasileira, utilizando-se outro instrumento para mensuração da QV
$(\mathrm{SF}-36)^{19}$. Embora a UFBA seja uma universidade pública, os alunos têm gastos com alimentação, fotocópias, transporte e compra de livros. Aqueles com maior renda familiar devem ter menos preocupações econômicas, possuem carro e podem adquirir livros, dependendo menos da biblioteca para seus estudos, o que deve proporcionar maior tranquilidade e, portanto, melhor qualidade de vida psicológica. Aqueles que não souberam informar qual a renda mensal aproximada de sua família apresentaram maior prevalência de baixa DPQV. É possível que a falta de conhecimento de sua renda familiar mensal aproximada seja reflexo de má interação familiar, falta de comunicação ou de organização financeira entre seus membros.

Os estudantes que referiram não ter dinheiro suficiente para suas despesas cotidianas apresentaram o dobro de prevalência de baixa DPQV. Estes resultados chamam a atenção para a influência que a questão econômica pode ter na vida do estudante e possivelmente em seu desempenho acadêmico e formação como um todo, apesar de estudar em uma universidade pública. Nesse contexto, a maioria dos estudantes precisa recorrer aos livros da biblioteca da sua faculdade, mas a maioria não está satisfeita com a qualidade dos livros disponíveis. Livros antigos, desatualizados e com má conservação podem dificultar o estudo e o rendimento acadêmico do aluno, o que pode explicar a maior prevalência de baixa DPQV encontrada no grupo insatisfeito.

A insatisfação com o custo das refeições disponíveis no ambiente da faculdade também se relacionou com alta prevalência de baixa DPQV. Como o curso de Medicina conta com aulas em vários turnos, a maioria dos alunos precisa fazer as refeições em ambientes próximos à faculdade, enquanto uma minoria costuma trazer comida de casa para a faculdade. Como o campus de Medicina não disponibiliza restaurante universitário e a maioria dos estudantes tem que recorrer a restaurantes terceirizados ou independentes, com preço mais elevado, o custo mensal com refeições é, muitas vezes, alto. Sobretudo para os estudantes com menor renda familiar, as despesas com alimentação podem pesar financeiramente e ser fonte de preocupação.

A alimentação não saudável foi relacionada com baixa DPQV no presente estudo. Assim como encontrado em estudo prévio feito com estudantes de Medicina de outra faculdade brasileira ${ }^{21}$, a maioria dos estudantes não considerou sua alimentação como saudável, e, entre estes, o maior motivo referido foi a falta de tempo. A maioria dos recém-ingressos, no entanto, considerou sua alimentação saudável, o que sugere que o ingresso na faculdade possa ser fator impor- 
tante na piora da alimentação desses indivíduos. Devido à indiscutível importância de uma alimentação saudável, esses resultados sugerem ser preciso dar maior atenção à questão, no intuito de possibilitar aos alunos acesso, preço e tempo disponível compatíveis com a manutenção de uma dieta balanceada.

A segurança na faculdade é outro ponto importante a ser considerado devido ao grande tempo passado pelos estudantes nesse ambiente. A necessidade de se deslocar constantemente entre os pavilhões de aula e nos arredores do campus exige maior atenção quanto à segurança dos alunos. A intranquilidade do aluno em relação à sua movimentação e permanência na faculdade e a sensação de perigo nesse ambiente devem ser um fator estressante e muitas vezes limitador de atividades. Isto pode explicar a insatisfação com a segurança no ambiente da faculdade encontrada como fator de aumento de prevalência de baixa DPQV.

Os motivos apresentados pelos estudantes para terem escolhido cursar Medicina foram variados, como aptidão, vivência de doenças, mercado de trabalho e vontade de ajudar os outros, entre outros. Foi possível separar os motivos em dois grupos principais: motivos relacionados a mercado de trabalho e questões financeiras, e motivos relacionados às aptidões. Aqueles que escolheram Medicina por motivos relacionados às aptidões e desejo de ajudar os outros apresentaram melhor DPQV. Os motivos apontados pelos estudantes neste estudo foram compatíveis com aqueles de estudos prévios, que mostraram que o desejo de ajudar os outros tem sido o principal motivo da escolha profissional ${ }^{24,31}$. No entanto, acredita-se que, não raramente, os estudantes têm sido impulsionados a escolher suas carreiras visando ao retorno financeiro, e entre as mais procuradas para este fim está a carreira médica. Independência financeira, influência familiar e status profissional ainda são motivações importantes e frequentes entre os que escolhem cursar Medicina ${ }^{24}$. Quando estes são os principais motivos da escolha, é possível que o estudante venha a se frustrar com o curso, o que pode explicar a menor DPQV encontrada nesse grupo. $\mathrm{O}$ cansaço e a frustração com o curso foram os motivos mais apontados pelos quase $40 \%$ de estudantes que já haviam pensado ou pensavam em abandoná-lo. Além disso, as dificuldades enfrentadas pelo médico em sua vida profissional atualmente e a redução do seu prestígio social tornam difícil para o estudante conciliar a ilusão construída com a realidade que experimenta ${ }^{32}$.

Como esperado, indivíduos que realizavam com frequência atividades prazerosas extra-acadêmicas apresentaram DPQV muito superior. Os alunos de Medicina mencionam como principais fontes de estresse a perda da liberdade pessoal, o excesso de pressões acadêmicas e a falta de tempo para o lazer, para estar com a família e com os amigos mais ínti$\operatorname{mos}^{33}$. A queixa de falta de tempo para atividades culturais, praticar esporte e estar com a família e amigos se coloca como questão central na discussão da qualidade de vida do estudante de Medicina ${ }^{21}$. A porcentagem de estudantes que disseram não desempenhar regularmente atividades diferentes das atividades acadêmicas e que lhes dessem prazer foi elevada e nitidamente superior àquela encontrada entre os recém-ingressos, sugerindo que fatores relacionados à faculdade são importantes nessa mudança. O curso de Medicina é longo e denso, restando pouco tempo livre para outras atividades de interesse do estudante. A abdicação de atividades prazerosas antes exercidas, como dança, academia ou curso de idiomas, por exemplo, pode ter um importante impacto no bem-estar do indivíduo. Estas atividades tornam-se muitas vezes necessárias para diminuir a tensão e a autocobrança relacionadas ao curso de Medicina. Além disso, momentos de interação social com família e amigos tendem a se tornar menos frequentes com a crescente demanda de tempo exigida pelo curso, inclusive nos fins de semana.

Em relação ao uso de substâncias psicoativas nos últimos seis meses, o álcool foi a mais frequente, seguido em ordem decrescente por maconha, tranquilizantes, anfetamina, lança-perfume e cocaína. Entretanto, em estudo prévio feito entre estudantes de Medicina de Salvador, a ordem das substâncias mais usadas nos últimos 12 meses foi: álcool, lança-perfume, tranquilizante e maconha ${ }^{34}$; já a frequência encontrada em estudo feito com estudantes de Medicina em São Paulo seguiu a ordem: álcool, solventes, maconha, tranquilizantes e cocaína ${ }^{35}$, mostrando que as proporções de uso dessas substâncias podem variar, embora o álcool se mantenha sempre na primeira posição. Em relação à proporção do uso de substâncias psicoativas por estudantes de Medicina, estudo realizado nos Estados Unidos verificou proporções de uso na vida de $98 \%$ para o álcool, $66 \%$ para a maconha, $55 \%$ para o tabaco, $32 \%$ para a cocaína, $22 \%$ para anfetaminas e $19 \%$ para tranquilizantes $^{36}$. Estas porcentagens são muito maiores do que as encontradas em estudos nacionais. Em estudantes de Medicina de São Paulo, a frequência de uso de substâncias psicoativas esteve relacionada a religião, renda familiar e tempo livre nos dias úteis ${ }^{37}$.

A ingestão de álcool com frequência pelo menos mensal nos últimos 12 meses se mostrou fator de aumento de prevalência de alta DPQV se comparada à ausência total de uso de bebida alcoólica nesse período. É possível que a ingestão não 
excessiva de álcool seja acompanhada de momentos prazerosos, de interação social, e, assim, um consumo periódico de bebidas alcoólicas seria reflexo da frequência desses momentos. Desse modo, o caráter socializador do uso de bebida alcoólica, além da sensação de relaxamento resultante da ação da própria substância, pode ajudar o indivíduo a aliviar suas tensões e se refletir em melhor QV. Por outro lado, o uso passado ou atual de drogas ilícitas mostrou-se fator de aumento de prevalência de baixa DPQV. A busca por drogas pode ser impulsionada pelo enfrentamento de problemas, sendo consequência de baixa DPQV, e também pode contribuir como causa de baixa $\mathrm{QV}$, devido aos efeitos diretos das próprias substâncias usadas e à potencial preocupação em relação ao seu caráter ilícito. Em estudo prévio feito entre estudantes de Medicina de Salvador, em 2005, a diversão foi apontada como a razão mais importante para o uso de substâncias psicoativas, a busca de relaxamento como segunda principal razão, e a fuga do estresse como terceira. Ainda nesse estudo, a maioria dos entrevistados considerou que o modo de vida do estudante de Medicina favorecia o uso de substâncias psicoativas $^{34}$.

Entre as limitações do estudo transversal, as seguintes foram identificadas no presente estudo: (a) vulnerabilidade ao viés da simultaneidade, não permitindo uma definição da sequência temporal entre os eventos estudados; foi possível identificar muitas variáveis que tanto poderiam causar como serem causadas pela baixa DPQV; (b) as características do estudante no momento da aplicação do questionário podem ter sido modificadas recentemente, podendo não refletir a realidade predominante em momento anterior ou mesmo na maior parte da sua vida acadêmica; isto pode ter sido minimizado pelo fato de algumas variáveis terem sido questionadas considerando-se a ocorrência de certo evento em determinado período de tempo no passado ("uso de álcool nos últimos 12 meses", por exemplo); (c) a razão de prevalências não é a melhor medida de associação para investigação etiológica; (d) o viés de prevalência pode ocorrer nos estudos transversais, especialmente ao se investigarem doenças muito agudas ou letais, os agravos sazonais e as de remissão periódica; no presente estudo, muito provavelmente, esse viés não ocorreu, pois o problema de saúde de interesse tem caráter crônico; contudo, este viés pode ter ocorrido porque não foram ativamente buscados os estudantes que não foram encontrados no momento de aplicação do questionário na turma à qual pertenciam, sendo que estes poderiam estar afastados por algum problema relacionado à saúde mental; avaliou-se, entretanto, que, como estas perdas foram pequenas, não introduziram viés significativo no estudo.

\section{CONCLUSÃO}

Os resultados sugerem que a vivência do curso de Medicina tem influência relevante na dimensão psicológica da qualidade de vida dos estudantes. Diante disso, admite-se a importância de dar atenção aos possíveis fatores de aumento de prevalência de baixa qualidade de vida dessa população, alguns dos quais foram evidenciados neste estudo, ressaltando-se aqueles relacionados à infraestrutura e organização do curso, por serem passiveis de soluções objetivas. Estudos em diferentes faculdades e períodos, realizados por outros pesquisadores, são necessários para melhor avaliação da influência do curso e da faculdade de Medicina na qualidade de vida dos estudantes e sua consequência na formação desses futuros profissionais. O conhecimento dessas condições de aprendizado e de suas consequências poderá orientar ações que visem modificar os fatores relacionados à faculdade que interferem negativamente na DPQV dos estudantes.

\section{REFERÊNCIAS}

1. Fleck MPA, (org.). A avaliação da qualidade de vida: guia para profissionais da saúde. Porto Alegre: Artmed; 2008.

2. Rameix S. Justifications et difficultés éthiques du concept de qualité de vie. Revue Prevenir. 1997;33:89-103.

3. Kluthcovsky ACGC, Kluthcovsky FA. O WHOQOL-bref, um instrumento para avaliar qualidade de vida: uma revisão sistemática. Rev Psiquiatr Rio Gd Sul. 2009;31(3):1-12. DOI: 10.1590/S0101-81082009000400007

4. Villanueva T, Haivas I. Studying medicine and quality of life. Student. BMJ 2006;14:170-1.

5. Almeida AM, Godinho TM, Bitencourt AGV, Teles MS, Silva AS, Fonseca DC, et al. Common mental disorders among medical students. J Bras Psiquiatr. 2007;56(4):24551. DOI: $10.1590 /$ S0047-20852007000400002

6. Lima MCP, Domingues MS, Cerqueira ATAR. Prevalência e fatores de risco para transtornos mentais comuns entre estudantes de medicina. Rev Saúde Pública. 2006;40:103541. DOI: 10.1590/S0034-89102006000700011

7. Chehuen Neto JA, Sirimarco MT, Pittondo MS, Sant'Ana Marques F, Baratti AB. Qualidade de vida dos estudantes de medicina e direito. HU Revista. 2008;34(3):197-203.

8. Cavestro JM, Rocha FL. Prevalência de depressão entre estudantes universitários. J Bras Psiquiatr. 2006;55(4):264-7. DOI: 10.1590/S0047-20852006000400001

9. Costa EFO, Andrade TM, Silvany Neto AM, Melo EV, Rosa ACA, Alencar MA, et al. Common mental disorders among medical students at Universidade Federal de Sergipe: a cross-sectional study. Rev Bras Psiquiatr. 2012 Mar;32(1):11-9 DOI: 10.1590/S1516-44462010000100005 
10. Cardoso HC, Bueno FCC, Mata JC, Alves APR, Jochims I, Vaz Filho IHR, et al. Avaliação da qualidade do sono em estudantes de Medicina. Rev Bras Educ Med. 2009;33:34955. DOI: $10.1590 /$ S0100-55022009000300005

11. Abraão CB, Coelho EP, Passos LBS. Prevalência de sintomas depressivos entre estudantes de medicina da Universidade Federal de Uberlândia. Rev Bras Educ Med. 2008;32(3):315-23. DOI: 10.1590/S0100-55022008000300006.

12. Almondes KMd, Araújo JFd. Padräo do ciclo sono-vigília e sua relaçäo com a ansiedade em estudantes universitários. Estud Psicol (Natal). 2003;8(1):37-43. DOI: 10.1590/S1413$-294 X 2003000100005$

13. Kerr-Corrêa F, Andrade AG, Bassit AZ, Boccuto NMVF. Uso de álcool e drogas por estudantes de medicina da Unesp. Rev Bras Psiquiatr. 1999;21:95-100. DOI: 10.1590/ S1516-44461999000200005

14. Aguiar SM, Vieira APGF, Vieira KMF, Aguiar SM, Nóbrega JO. Prevalência de sintomas de estresse nos estudantes de medicina. J Bras Psiquiatr. 2009;58(1):34-8. DOI: 10.1590/ S0047-20852009000100005

15. Meleiro AMAS. Suicídio entre médicos e estudantes de medicina. Rev Assoc Med Bras. 1998;44:135-40. DOI: 10.1590/ S0104-42301998000200012

16. Rimmer J, Halikas JA, Schuckit MA. Prevalence and incidence of psychiatric illness in college students: a four year prospective study. J Am Coll Health. 1982;30(5):207-11.

17. Hosmer D, Lemeshow S. Applied logistic regression. $2^{2}$ ed. New York: Wiley - Interscience; 2000.

18. Minayo MCS, Hartz ZMA, Buss PM. Qualidade de vida e saúde: um debate necessário. Ciênc Saúde Coletiva. 2000;5:7-18. DOI: 10.1590/S1413-81232000000100002

19. Paro HB, Morales NM, Silva CH, Pinto RM, Morales RR, Mendonça TM, et al. Health-related quality of life of medical students. Med Educ. 2010;44(3):227-35. DOI: 10.1111/j.1365-2923.2009.03587.x

20. Guthrie E, Black D, Bagalkote H, Shaw C, Campbell M, Creed F. Psychological distress and burnout in medical students: A five-year prospective longitudinal study. J R Soc Med. 1998;91(5):237-43.

21. Fiedler PT. Avaliação da qualidade de vida do estudante de medicina e da influência exercida pela formação acadêmica. São Paulo; 2008. Doutorado [Tese] - Universidade de São Paulo.

22. Hafferty F. Beyond curriculum reform: confronting medicine's hidden curriculum. Acad Med. 1998 Apr;73(4):403-7.

23. Moffat KJ, McConnachie A, Ross S, Morrison JM. First year medical student stress and coping in a problem-based learning medical curriculum. Med Educ. 2004;38(5):482-91.
24. Moreira SNT, Nogueira e Silva CA, Tertulino FF, Tertulino FMF, Vilar MJP, Azevedo GD. Processo de significação de estudantes do curso de medicina diante da escolha profissional e das experiências vividas no cotidiano acadêmico. Rev Bras Educ Med. 2006;30:14-9. DOI: 10.1590/S010055022006000200003

25. Compton M, Carrera J, Frank E. Stress and depressive symptoms/dysphoria among US medical students: results from a large, nationally representative survey. J Nerv Ment Dis. 2008;196(12):891-7.

26. Dahlin M, Joneborg N, Runeson B. Stress and depression among medical students: a cross-sectional study. Med Educ. 2005;39(6):594-604.

27. Hojat M, Glaser K, Xu G, Veloski J, Christian E. Gender comparisons of medical students' psychosocial profiles. Med Educ. 1999 May;33(5):342-9.

28. Dyrbye LN, Thomas MR, Huschka MM, Lawson KL, Novotny PJ, Sloan JA, et al. A multicentre study of burnout, depression, and quality of life in minority and non-minority US medical students. Mayo Clin Proc. 2006;81(11):1435-42.

29. Baldassin S, Alves TCTF, Andrade AG, Martins AN. The characteristics of depressive symptoms in medical students during medical education and training: a cross-sectional study. BMC Med Educ. 2008;8:60. DOI: 10.1186/1472-6920-8-60

30. Allardt E. A welfare model for selecting social indicators of national developmente. Policy Sciences. 1973;4:64-74. DOI: 10.1007/BF01404933

31. Mascaretti L. Perfil do aluno da faculdade de Medicina de São Paulo. Rev Bras Educ Med. 2002;26:55.

32. Arruda PCV, Millan LR. A vocação médica. In: Millan LR, De Marco OLN, Rossi E, Arruda PCV. O universo psicológico do futuro médico. São Paulo: Casa do psicólogo; 1999. p. 15-29.

33. Millan LR, Rossi E, De Marco OLN. A psicopatologia do estudante de Medicina. In: Millan LR, De Marco OLN, Rossi E, Arruda PCV. O universo psicológico do futuro médico. São Paulo: Casa do psicólogo; 1999. p. 83-101.

34. Lemos KM, Neves NMBC, Kuwano AY, Tedesqui G, Bittencourt AGV, Neves FBCS, et al. Uso de substâncias psicoativas entre estudantes de Medicina de Salvador (BA). Rev Psiq Clín. 2007;34(3):118-24.

35. Andrade AG, Bassit AZ, Kerr-Corrêa F, Tonhon AA, Boscovitz EP, Cabral M, et al. Fatores de risco associados ao uso de álcool e drogas na vida, entre estudantes de medicina do Estado de Säo Paulo. Rev ABP-APAL. 1997;19:117-26.

36. Baldwin Jr. DC, Hughes PH, Conard SE, Storr CL, Sheehan DV. Substance use among senior medical students, a survey of 23 medical schools. JAMA. 1991;265(16):2074-8. 
37. Silva LVER, Malbergier A, Stempliuk VA, Andrade AG. Fatores associados ao consumo de álcool e drogas entre estudantes universitários. Rev Saúde Pública. 2006;40:280-8. DOI: 10.1590/S0034-89102006000200014

\section{CONTRIBUIÇÃO DOS AUTORES}

Sofia Senna Gonçalves, contribuiu para este trabalho especificamente como planejamento, aplicação de questionários, digitação e análise dos dados, interpretação e redação do artigo. Annibal Muniz Silvany Neto, contribuiu para este trabalho especificamente como planejamento, análise dos dados, interpretação e redação do artigo
CONFLITO DE INTERESSES

Declarou não haver.

\section{ENDEREÇO PARA CORRESPONDÊNCIA}

Sofia Senna Gonçalves

Rua Irmã Dulce, nº 250 - apto. 004

Brotas - Salvador

CEP. 40286-030 BA

E-mail: sosenna@hotmail.com 